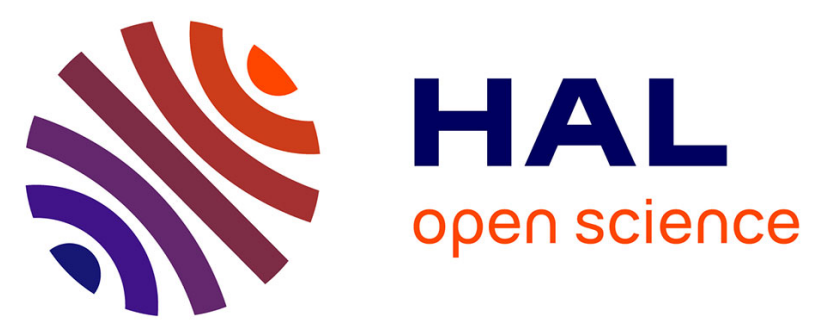

\title{
A mixed-effects model with time reparametrization for longitudinal univariate manifold-valued data
}

Jean-Baptiste Schiratti, Stéphanie Allassonniere, Alexandre Routier, Olivier Colliot, Stanley Durrleman

\section{- To cite this version:}

Jean-Baptiste Schiratti, Stéphanie Allassonniere, Alexandre Routier, Olivier Colliot, Stanley Durrleman. A mixed-effects model with time reparametrization for longitudinal univariate manifold-valued data. IPMI - Information Processing in Medical Imaging, Sebastien Ourselin, Daniel C. Alexander, Carl-Fredrik Westin, M. Jorge Cardoso, Jun 2015, Sleat, Isle of Skye, United Kingdom. pp.564-575. hal-01163213

\section{HAL Id: hal-01163213 \\ https://hal.science/hal-01163213}

Submitted on 12 Jun 2015

HAL is a multi-disciplinary open access archive for the deposit and dissemination of scientific research documents, whether they are published or not. The documents may come from teaching and research institutions in France or abroad, or from public or private research centers.
L'archive ouverte pluridisciplinaire HAL, est destinée au dépôt et à la diffusion de documents scientifiques de niveau recherche, publiés ou non, émanant des établissements d'enseignement et de recherche français ou étrangers, des laboratoires publics ou privés. 


\title{
A mixed-effects model with time reparametrization for longitudinal univariate manifold-valued data
}

\author{
J.-B. Schiratti ${ }^{3,1}$, S. Allassonnière ${ }^{3}$, A. Routier ${ }^{1}$, the Alzheimer's Disease \\ Neuroimaging Initiative, O. Colliot ${ }^{1,2}$, S. Durrleman ${ }^{1}$ \\ 1 Inria Paris-Rocquencourt, Inserm U1127, CNRS UMR 7225, Sorbonne Universités, \\ UPMC Univ Paris 06 UMRS 1127, Institut du Cerveau et de la Moelle épinière, ICM, \\ F-75013, Paris, France \\ 2 AP-HP, Pitié-Salpêtrière Hospital, Departments of Neurology and Neuroradiology, \\ F-75013, Paris, France \\ 3 Ecole Polytechnique, Palaiseau, France
}

\begin{abstract}
Mixed-effects models provide a rich theoretical framework for the analysis of longitudinal data. However, when used to analyze or predict the progression of a neurodegenerative disease such as Alzheimer's disease, these models usually do not take into account the fact that subjects may be at different stages of disease progression and the interpretation of the model may depend on some implicit reference time. In this paper, we propose a generative statistical model for longitudinal data, described in a univariate Riemannian manifold setting, which estimates an average disease progression model, subject-specific time shifts and acceleration factors. The time shifts account for variability in age at disease-onset time. The acceleration factors account for variability in speed of disease progression. For a given individual, the estimated time shift and acceleration factor define an affine reparametrization of the average disease progression model. This statistical model has been used to analyze neuropsychological assessments scores and cortical thickness measurements from the Alzheimer's Disease Neuroimaging Initiative database. The numerical results showed that we can distinguish between slow versus fast progressing and early versus late-onset individuals.
\end{abstract}

\section{Introduction}

The Alzheimer's disease (AD), and most neurodegenerative diseases alike, is a slowly progressive neurodegenerative disorder and a growing public health issue as the number of people diagnosed with $\mathrm{AD}$ is steadily increasing over the years, partly due to an increasing ageing population. The progressive loss of cognitive functions, such as memory, language and reasoning, are the observable consequences of pathological processes which started to affect the brain several years before. In [1], Clifford R. Jack et al. proposed a widely accepted hypothetical model which describes the temporal evolution of the AD biomarkers in which the decline in cognitive functions and memory loss occur in the late stages of 
the disease. The impairment of the cognitive functions is preceded by decreasing cerebrospinal fluid (CSF) amyloid- $\beta 42\left(A \beta_{1-42}\right)$ peptide, increasing CSF tau protein and neurodegeneration, characterized by cerebral atrophy and neuron loss. In order to increase the chances of success of a given treatment, the need for an early diagnosis and the need to understand the progression of the disease are crucial.

Longitudinal data, which consists in repeated observations of a given individual or group of individuals over time, allows to study the progression of a disease and the statistical analysis of longitudinal data may provide useful informations to help diagnose AD in its early phase. Mixed effects models provide a rich framework to analyze longitudinal measurements. The fixed (and respectively random) effects allow to describe the model at the group (respectively subject) level and the distribution of the random effects accounts for inter-subject variability. A particular case of the linear mixed-effects models introduced by Laird and Ware in [5] can be used to model a linear relationship between univariate longitudinal measurement and the times of observation : the random slope and intercept model. If $y_{i, j}$ denotes the $j$-th observation of the $i$-th subject associated to time $t_{i, j}$, the random slope and intercept model would write $: y_{i, j}=\left(\bar{a}+a_{i}\right)\left(t_{i, j}-t_{0}\right)+\left(\bar{b}+b_{i}\right)+\varepsilon_{i, j}$, where $\varepsilon_{i, j}$ denotes a Gaussian noise with zero mean. The fixed effects $\bar{a}$ and $\bar{b}$ respectively correspond to the slope and intercept of an average trajectory $\bar{D}(t)=\bar{a}\left(t-t_{0}\right)+\bar{b}$ whereas $t_{0}$ plays the role of a reference time. The subject-specific slope $a_{i}$ and $b_{i}$ correspond to the slope and intercept of the trajectory of the $i$-th individual.

If the longitudinal observations arise, for example, from developmental studies, breeding studies or pharmacological studies, there is a natural choice for the baseline time $t_{0}$, which may represent the time of birth, beginning of the study or time at which a certain drug has been administered. In those situations, the baseline time is known and is therefore not estimated along with the parameters of the model. However, in studies on neurodegenerative diseases, such as Alzheimer's disease, there may be no natural choice for the reference time. As pointed out by Yang et al. in [4] and Delor et al. in [3], the disease-onset time is probably different for every individual and, at a given age, two individuals may be at very different stages of disease progression. When the baseline (or disease-onset) time is unknown, one may consider it as a fixed effect of the given model. In [2], the authors proposed an interesting mixed-effect model for scalar measurements but the model did not account for the difference in stages progression among individuals. Yang et al. [4] and Delor et al. [3] addressed this issue by including time shifts in their models but Yang et al. did not estimate the time shifts in a statistical framework. In Delor et al., the observations of a given individual were shifted in time before the estimation of a disease-onset time. In both situations, the reference time was not estimated. In [8], Singh et al. proposed an interesting model for longitudinal manifold-valued observations using parallel transport. However, if the model is written for univariate longitudinal data, the model does include a random effect to account for the variability in speed of disease progression. 
When the time reference time $t_{0}$ is considered unknown, the linear model given above is not identifiable and there is a relation between the distribution of the intercept random effect $b_{i}$ and the fixed effect $t_{0}$, which makes the interpretation of the distribution of the intercept random effect $b_{i}$ difficult.

To address the issues we pointed out, we propose to replace the intercept random effect $b_{i}$, which characterizes the distribution of the measurement values at time $t_{0}$, by a time shift $\tau_{i}$ which measures the delay (or advance) in disease progression. We therefore consider the model $: y_{i, j}=v_{0} \alpha_{i}\left(t_{i, j}-t_{0}-\tau_{i}\right)+\varepsilon_{i, j}$ where $\alpha_{i}$ is a random effect log-normally distributed with mean equal to 1 and $\tau_{i}$ is the time shift random effect normally distributed with mean 0 . The fixed effect $v_{0}$ correspond to the slope of the mean trajectory and is the fixed effect associated to $\alpha_{i}$ whereas $t_{0}$ is now the fixed effect associated to the random effect $\tau_{i}$. If $D$ denotes the straight line $D(t)=v_{0}\left(t-t_{0}\right)$, the model writes $: y_{i, j}=$ $D\left(\alpha_{i}\left(t_{i, j}-t_{0}-\tau_{i}\right)+t_{0}\right)+\varepsilon_{i, j}$. As a consequence, the random effects $\alpha_{i}$ and $\tau_{i}$ allow to define a subject-specific affine reparametrization of the straight line $\mathrm{D}$, which can be thought of as an average trajectory. The acceleration factor $\alpha_{i}$ and the time shift $\tau_{i}$ of the affine subject-specific reparametrization provide informations on the dynamical evolution of a given subject with respect to the average model: the acceleration factor will determine whether an individual is progressing faster than the individual trajectory. The time shift determines whether the individual is lagging behind or ahead of the average model. Therefore, the random effects of the model will allow to determine, among a group of individuals, who are fast or slow progressing individuals and whether a given individual might develop the disease earlier (early-onset) or later (late-onset) than the estimated reference time $t_{0}$.

Moreover, since the subject-specific trajectories are affine reparametrizations of an average trajectory, this model can easily be generalized to longitudinal measurements on a Riemannian manifold. The average trajectory may be replaced with a geodesic of the manifold and the subject-specific trajectories will remain geodesics on the manifold. As a matter of fact, longitudinal measurements arising from neuroimaging, neuropsychological tests or clinical examinations may belong to nonlinear spaces. The scores to a neuropsychological test often belong to a bounded interval. Riemannian manifolds offer a very flexible framework which includes the previous examples. The generalization of this statistical model to Riemannian manifold-valued observations could be used either to analyze shapes, cortical thickness measurements or neuropsychological assessment scores. After a proper renormalization, the ADAS-Cog 13 scores belong to the open unit inverval $(0,1)$ which, equipped with a specific metric, can be seen as a univariate Riemannian manifold. For this metric, the geodesics are logistic curves. The models presented in [2] and [3] do not generalize to manifold-valued data. In [9], Lorenzi et al. used Riemannian manifold techniques to estimate a model of the brain's normal ageing from healthy individuals T1 MR scans. The model was used to compute a time shift, called morphological age shift, which corresponds to the actual anatomical age of the subject with respect to an estimated average age for healthy subjects. However, the subject-specific time shifts 
were not estimated as parameters of a statistical model. In [7], Datar et al. generalized a particular linear mixed-effects model to longitudinal shape analysis but the proposed model did not include time shifts to account for the variability in stages of disease progression among the population. Even though the model is used to analyze shapes (given by a set of points), the model is not described in a Riemannian manifold setting.

The work presented herein will generalize the model written above to a Riemannian manifold included in $\mathbb{R}$. In Section 2, we will introduce our model and we will present two particular cases of this model : the straight lines model and the logistic curves model. We also explain how the parameters of the model are estimated. In Section 3, the straight lines model and logistic curves model were used to analyze longitudinal ADAS-Cog and cortical thickness measurements from the ADNI database. We show that the estimated random effects of the model allowed to distinguish between slow versus fast progressing individuals and early versus late-onset individuals.

\section{A mixed-effects model with time reparametrization for manifold-valued observations}

\subsection{Model description}

Let us assume that we observe $p$ different individuals. For each individual, we have $n_{i}$ observations, obtained at times $t_{i, 1}<\ldots<t_{i, n_{i}}$ and the observations of the $i$-th individual are denoted by $y_{i, 1}, \ldots, y_{i, n_{i}}$. We assume that each observation $y_{i, j}$ is a point on a one-dimensional Riemannian manifold $(M, g)$ included in $\mathbb{R}$. In addition to this, we assume that the geodesics of $M$ are defined on the entire real line.

Let $p_{0}$ be a point in $M, t_{0}$ in $\mathbb{R}$ and $v_{0} \in T_{p_{0}} M$, a tangent vector to $M$ at the point $p_{0}$. The triplet $\left(p_{0}, t_{0}, v_{0}\right)$ allows to define an average trajectory $\gamma_{p_{0}, t_{0}, v_{0}}$, the geodesic of $M$ which passes through the point $p_{0} \in M$ at time $t_{0}$ and with the velocity $v_{0} \in T_{p_{0}} M \simeq \mathbb{R}$. This average trajectory summarises the progression of all the individuals and could be interpreted as the evolution of an "average subject". While the average subject passes through the point $p_{0}$ at time $t_{0}$ with the velocity $v_{0}$, we assume that the trajectory of the $i$-th subject is the geodesic $\gamma_{i}$ which passes through the point $p_{0}$ at time $t_{0}+\tau_{i}$ with velocity $\alpha_{i} v_{0}$. The parameters $\left(p_{0}, t_{0}, v_{0}\right)$ will be the fixed effects of the model whereas $\alpha_{i}$ and $\tau_{i}$ will be the random effects of the model. The point $p_{0}$ in $M$ can be understood as an observation point. A simplistic interpretation of the role played by $\alpha_{i}$ and $\tau_{i}$ is the following : an observer standing at the point $p_{0}$ in $M$ and aware that the average subject passes through the point at the time $t_{0}$, with velocity $v_{0}$, will be able to tell whether the $i$-th subject passes through this point earlier or later than the average subject and whether he passes faster or slower. More precisely, $\tau_{i}$ is a time shift which will provide an information on whether the progression of the $i$-th trajectory is ahead of the average trajectory or not 
and $\alpha_{i}$ provides the information on whether the $i$-th subject is evolving faster or slower than the average subject. As a consequence, the random effects $\alpha_{i}$ and $\tau_{i}$ take into account the fact that two different subjects may be at very different stages of disease progression and allow for inter-subjects comparaisons. The description above makes the assumption that every subject-specific trajectory of disease progression will eventually reach a common value $p_{0}$. This assumption does not make sense in a higher-dimensional Riemannian manifold. In order to generalize the proposed model to a higher-dimensional setting, one would need to add another random effect, associated to the fixed effect $p_{0}$.

For a point $p_{0} \in M$ and $t_{0} \in \mathbb{R}, v_{0} \in T_{p_{0}} M$, we define $\gamma_{p_{0}, t_{0}, v_{0}}:=\operatorname{Exp}_{t_{0}, p_{0}}\left(\alpha_{i} v_{0}\right)(\cdot)$ as the geodesic which passes through the point $p_{0}$ at time $t_{0}$ with velocity $v_{0}$ $: \gamma_{p_{0}, v_{0}, t_{0}}$ is the unique curve, drawn on $M$ and defined on $\mathbb{R}$, which satisfies $\nabla_{\dot{\gamma}_{p_{0}, t_{0}, v_{0}}} \dot{\gamma}_{p_{0}, t_{0}, v_{0}}=0$ and $\gamma_{p_{0}, t_{0}, v_{0}}\left(t_{0}\right)=p_{0}, \dot{\gamma}_{p_{0}, t_{0}, v_{0}}\left(t_{0}\right)=v_{0}$. For the dataset $\left(t_{i, j}, y_{i, j}\right)\left(1 \leq i \leq p\right.$ and $\left.1 \leq j \leq n_{i}\right)$, we assume the following model :

$$
y_{i, j}=\operatorname{Exp}_{t_{0}+\tau_{i}, p_{0}}\left(\alpha_{i} v_{0}\right)\left(t_{i, j}\right)+\varepsilon_{i, j}
$$

where $\alpha_{i}=\exp \left(\eta_{i}\right)$ and :

$$
\left\{\begin{array}{l}
\eta_{i} \sim \otimes_{i=1}^{p} \mathcal{N}\left(0, \sigma_{\eta}^{2}\right) \\
\tau_{i} \sim \otimes_{i=1}^{p} \mathcal{N}\left(0, \sigma_{\tau}^{2}\right) \\
\varepsilon_{i, j} \sim \otimes_{i, j} \mathcal{N}\left(0, \sigma^{2}\right) .
\end{array}\right.
$$

The random variables $\left(\eta_{i}\right)_{1 \leq i \leq p}$ and $\left(\tau_{i}\right)_{1 \leq i \leq p}$ are assumed independent. The vector $\theta=\left(p_{0}, t_{0}, v_{0}, \sigma_{\eta}, \sigma_{\tau}, \sigma\right)^{\top}$ is the vector of the parameters of the model. We will discuss later how this vector is estimated from the data.

An important feature of the model (1) is that in can be written as follows :

$$
\begin{aligned}
y_{i, j} & =\gamma_{p_{0}, t_{0}, v_{0}}\left(\alpha_{i} v_{0}\left(t_{i, j}-t_{0}-\tau_{i}\right)\right)+\varepsilon_{i, j} \\
& =\gamma_{i}\left(t_{i, j}\right)+\varepsilon_{i, j} .
\end{aligned}
$$

The subject-specific trajectory of the $i$-th subject, the geodesic $\gamma_{i}$, therefore appears as an affine reparametrization of the average trajectory $\gamma_{p_{0}, t_{0}, v_{0}}$. As a consequence, in order to compare the progession of a given individual with respect to the average trajectory (respectively compare two given subjects together), one only need to compare the affine reparametrization $t \mapsto \alpha_{i} v_{0}\left(t-t_{0}-\tau_{i}\right)$ with the identity map $t \mapsto t$ (respectively, compare the slopes and intercepts of the two subject-specific affine reparametrization). We can observe that these reparametrization play the same role as the time warps which were introduced by Durrleman et al. in [11] and [10], in the context of longitudinal shape analysis. The time warps considered in those papers were diffeomorphisms of the real line which accounted for the different rates of shape changes and were constructed using the LDDMM framework : the diffeomorphism was obtained by integration of a time-varying vector field which belongs to a Gaussian reproducing kernel Hilbert space. The affine reparametrizations (with non-zero intercept) are also diffeomorphisms but only model a constant rate of progression. However, with 
only two random effects, we are able to describe the progression of a given subject in a realistic and easily interpretable manner.

Considering $p_{0}$ as a fixed effect of the model and estimating $p_{0}$ along with the other fixed effects ensures that the estimated $p_{0}$ will be the best observation point possible. It should be noted that the model does not depend on a reference time because if the observations $\left(t_{i, j}, y_{i, j}\right)$ are transformed into $\left(t_{i, j}+t_{0}^{\prime}, y_{i, j}\right)$ then, only the estimated value of $t_{0}$ will change whereas the variance parameters will remain the same. Therefore, the interpretation of the random effects (and between-subjects or subjects-average comparaisons) do not depend on the timeline.

\subsection{The straight lines model}

In this section, we will illustrate the flexibility of the model we introduced in the previous section. The choice of the Riemannian metric on $M$ determines the shape of the geodesics of $M$. By taking the canonical metric on $\mathbb{R}$, the geodesics will be straight lines.

When $M=\mathbb{R}$ is equipped with the canonical metric, $M$ is a geodesically complete Riemannian manifold and the geodesics of $M$ are of the form $t \mapsto$ $p+t v$. In this case, (1) writes:

$$
y_{i, j}=p_{0}+\alpha_{i} v_{0}\left(t_{i, j}-t_{0}-\tau_{i}\right)+\varepsilon_{i, j} .
$$

Even though the model is called "linear", (4) does not belong to the class of linear mixed-effects models introduced in [5].

\subsection{The logistic curves model}

The situation when $M=(0,1)$ may arise when the observations are naturally bounded. For example, neuropsychological assessments such as ADAS-Cog or MMSE produce positive scores which are bounded above by a maximum score. The 13 questions ADAS-Cog assessment test is marked out of 85 points. Therefore, a typical ADAS-Cog score is a number in $[0,85]$. By a proper renormalization, the score values can be considered as points in the interval $(0,1)$. Fitting a linear model to such observations might not be such a good idea since the fitted trajectories will not always remain in the domain $(0,1)$. On the other hand, $(0,1)$ corresponds to the range of logistic curves with asymptote equal to 1 . In order to equip the open interval $(0,1)$ with a Riemannian manifold structure, we must give a Riemannian metric. At a point $p_{0} \in(0,1)$, the tangent space $T_{p_{0}} M$ is isomorphic to $\mathbb{R}$. For all $p_{0}$ in $M$, we define $g_{p_{0}}$ to be the following inner product $: \forall(u, v) \in T_{p_{0}} M, g_{p_{0}}(u, v)=u M\left(p_{0}\right) v$ where $M\left(p_{0}\right)=\frac{1}{p_{0}^{2}\left(1-p_{0}\right)^{2}}$. The mapping $p_{0} \mapsto g_{p_{0}}$ is smooth on $M$ and defines a Riemannian metric on $M$. In this particular setting, the geodesic equation $\nabla_{\dot{\gamma}} \dot{\gamma}=0$ writes : $\ddot{\gamma}(t)+\Gamma_{1,1}^{1}(\gamma(t))(\dot{\gamma}(t))^{2}=0$ where $\Gamma_{1,1}^{1}$ is the Christoffel symbol determined by the only coefficient $g_{1,1}(t)=M(t)$ of the metric. The 
geodesic equation writes : $\ddot{\gamma}(t)-\left(\frac{1}{\gamma(t)}+\frac{1}{\gamma(t)-1}\right)(\dot{\gamma}(t))^{2}=0$, or equivalently : $\gamma(\gamma-1) \ddot{\gamma}-(2 \gamma-1)(\dot{\gamma})^{2}=0$. Solving for this differential equation allows to determine that the geodesics of $M$ are logistic curves of the form $t \mapsto \frac{1}{1+a \exp (-r t)}$ with $a>0$ and $r \in \mathbb{R}$. The geodesics are defined on $\mathbb{R}$ and therefore ensure that $M$ is a geodesically complete Riemannian manifold. In this case, (1) writes :

$$
y_{i, j}=\frac{1}{1+\left(\frac{1}{p_{0}}-1\right) \exp \left(-\frac{\alpha_{i} v_{0}}{p_{0}\left(1-p_{0}\right)}\left(t_{i, j}-t_{0}-\tau_{i}\right)\right)}+\varepsilon_{i, j} .
$$

\subsection{Estimation of the parameters of the model}

The satistical model described in (1) is a nonlinear mixed-effects model with gaussian random effects. Such models have been extensively studied in the litterature and several methods exist for maximum likelihood estimation. The estimation of the parameters $\left(p_{0}, t_{0}, v_{0}, \sigma_{\eta}, \sigma_{\tau}, \sigma\right)$ was coded into the SAS software (SAS Institute) using the Gauss-Hermite quadrature approximation with one quadrature point. This quadrature approximation method is equivalent to the Laplace approximation of the observed likelihood. During the estimation procedure, the objective function resulting from the Laplace approximation was minimized using the Newton-Raphson algorithm or the Nelder-Mead simplex algorithm. It should be mentioned that these estimation procedures were quite sensitive to the choice of starting values. However we observed that this method was more efficent and more robust than the Alternating Algorithm, proposed by Lindstrom and Bates in [13] or Pinheiro and Bates in [12], and implemented in other softwares.

\section{Results}

\subsection{Data}

We used data from the Alzheimer's Disease Neuroimaging Initiative (ADNI) database [15]. The ADAS-Cog scores we used were collected for 1391 individuals enrolled in the ADNI1, ADNIGO and ADNI2 phases and cortical thickness measurements for 725 individuals from the ADNI1 database. The longitudinal follow-up of these individuals ranges from 18 months to 4 years. Diagnoses were recorded for every individual and at each visit. These subject-specific sequences of diagnoses allowed to classify the individuals into 4 groups of interest : stable controls (329 individuals), stable mild cognitive impairment, also denoted as stable MCI (471 individuals), stable Alzheimer patients (248 individuals) and converters MCI (282 subjects). Cortical thickness measurements were available for 194 stable controls, 182 stable MCI, 170 converters MCI and 162 Alzheimer patients. The individuals who reverted to control or MCI were not included in these groups. 


\subsection{Experimental results}

The ADAS-Cog scores were normalized by 85, which corresponds to the maximum possible score, and analyzed using the logistic curves model. For each individual, a couple of random variables $\left(\alpha_{i}, \tau_{i}\right)$ where $\alpha_{i}=\exp \left(\eta_{i}\right)$ were estimated along with the parameters of the model. We recall that $\alpha_{i}$ (and respectively $\tau_{i}$ ) account for the change in speed of disease progression (and respectively delay in disease progression) of a given individual with respect to the average disease progression trajectory. The couples $\left(\eta_{i}, \tau_{i}\right)$ have been plotted in figure 1 .

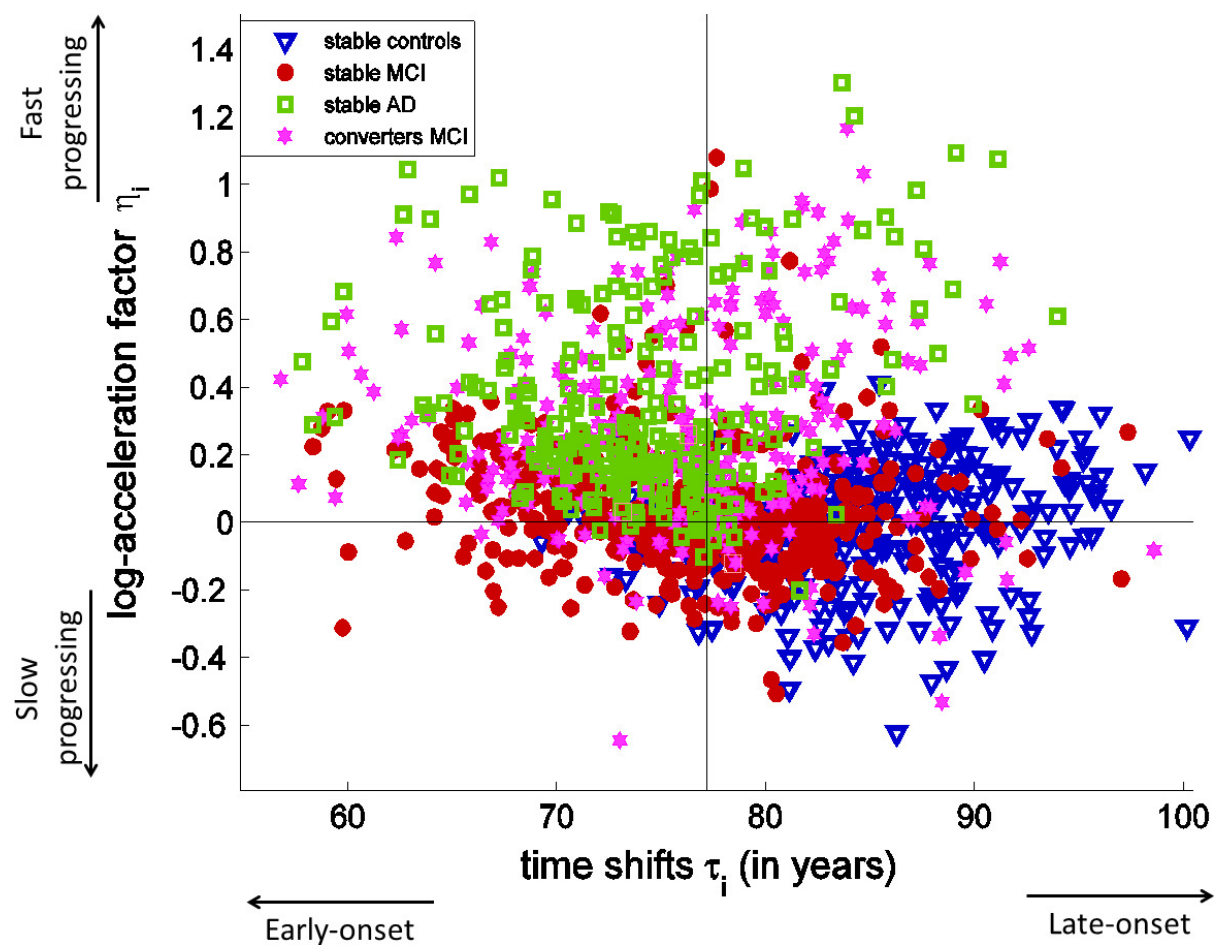

Fig. 1. Log-acceleration factors $\eta_{i}$ plotted against the time shifts $\tau_{i}$ for the 1391 individuals with ADAS-Cog measurements. An horizontal line was plotted at the level $\eta_{i}=0$ (no change in speed with respect to the average trajectory) and at $\tau_{i}=t_{0}=77.17$ (the estimated reference time $t_{0}$ ).

The results presented in figure 1 allow to compare the informations provided by $\left(\eta_{i}, \tau_{i}\right)$ across the groups of interest we previously mentioned. We can observe that stable controls have larger time shifts than other groups. The stable controls can be considered as late-onset individuals who are not, on average, evolving faster than the average disease progression trajectory. On the other hand, stable Alzheimer patients and MCI individuals tend to have smaller time 
shifts than stable controls. A portion of the stable MCI and most of the stable Alzheimer patients can be considered as early-onset individuals. It appears clearly that stable Alzheimer patients and converters MCI are fast progressing individuals even though a small number of converters MCI are slow-progressers. On average, stable MCI and stable controls are not progressing faster than the average disease progression scenario. These observations are coherent with the diagnoses of the individuals and the subject- specific random effects allow to distinguish groups of individuals which are coherent with their diagnoses. The disease progression for Alzheimer patients and converters MCI is faster and started earlier than for stable controls. The Alzheimer patients are quite clearly separated from the stable controls. In the model, the random effects $\eta_{i}$ and $\tau_{i}$ are assumed independent, therefore uncorrelated. For this experiment, we computed the estimated covariance matrix of the random effects. For stable controls, we find that $\operatorname{Corr}\left(\eta_{i}, \tau_{i}\right)=0.20$, for stable MCI : $\operatorname{Corr}\left(\eta_{i}, \tau_{i}\right)=-0.17$, for stable $\mathrm{AD}$ $: \operatorname{Corr}\left(\eta_{i}, \tau_{i}\right)=0.12$ and for converters MCI $: \operatorname{Corr}\left(\eta_{i}, \tau_{i}\right)=0.18$. These results seem coherent with the shape of the point clouds in the log-acceleration-timeshift plane, especially for stable controls and stable MCI.

The cortical thickness measurements (computed with Freesurfer) were averaged within 34 regions of interest defined with the Desikan-Killiany [19] cortical atlas. The averaged cortical thickness measurements were analyzed using the straight lines model. For each subject, time shifts and acceleration factors were estimated and the corresponding values were displayed on the cortical surface. The differences in the estimated time shifts and acceleration factors were compared between Alzheimer patients and controls (figure 2), and between converters MCI and stable MCI (figure 3). Significance level was set at 0.05, corrected for multiple comparisons using Bonferroni correction. Alzheimer patients present accelerated gray matter loss compared to stable controls in a large number of regions, with highest speed in temporal (including entorhinal cortex, parahippocampal gyrus, superior and middle temporal gyri), parietal associative (including precuneus) and frontal regions. A similar topographical pattern was found for converters MCI compared to stable MCI but with smaller accelerations than in $\mathrm{AD}$ patients. On the contrary, primary motor and sensitive as well as visual cortices were spared. These results are highly consistent with the spatial-temporal progression patterns of neurodegeneration evidenced in histopathological studies [16], [17]. Furthermore, accelerated atrophy has also been recently shown to coincide with disease-onset [18]. On the other hand, the estimated time shifts were not significantly different for the vast majority of regions. In the few significant regions, the magnitude of the time-shifts was small. This is in contrast with the large time-shift values found for the cognitive variables. This can be attributed to the slow but consistent age-related atrophy that is present in control subjects.

\section{Discussion and perspectives}

We proposed a statistical model which allows to model the progression of a neurodegenerative disease for a group of individuals. The power of our approach 

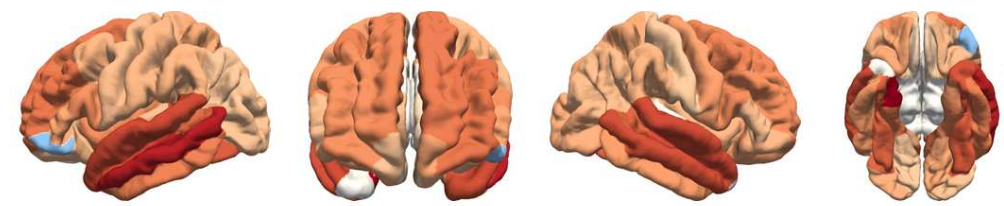

Difference of acceleration factors
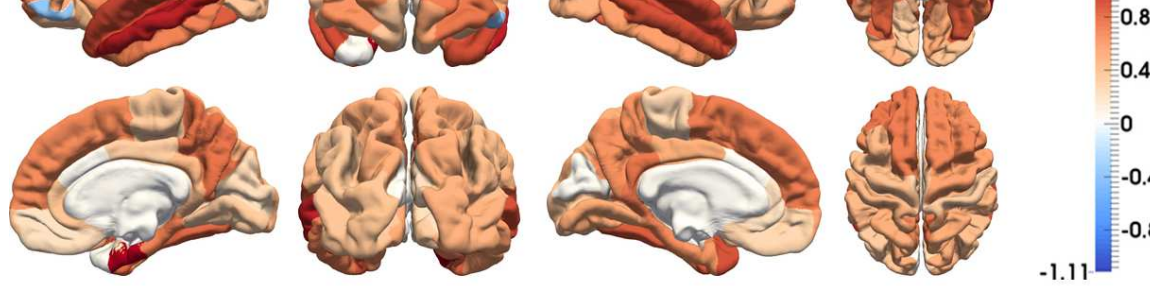

0.4

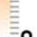

0
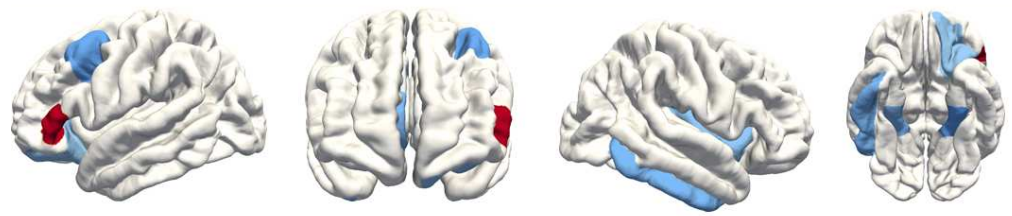

Difference of

time shifts

3.11
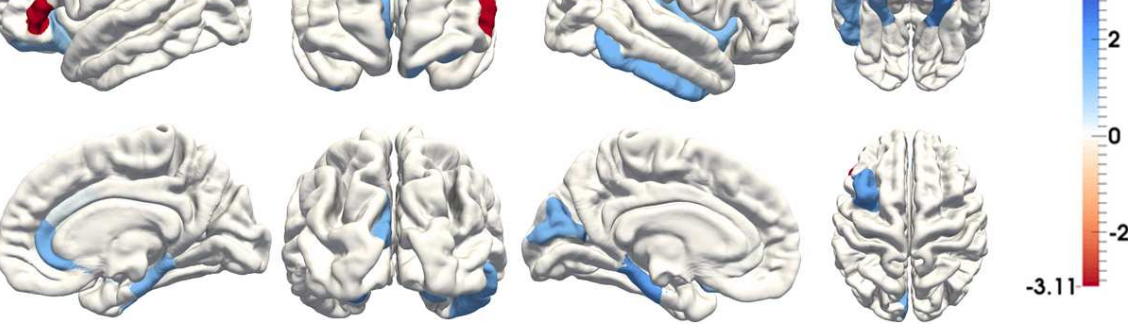

0

Fig. 2. At the top (respectively bottom) : the difference in averaged acceleration factors (respectively time shifts) between AD patients and stable controls is displayed on the cortex. Acceleration factors (and respectively time shifts) were averaged per regions of interest. Only regions where the difference was statistically significant $(p<0.05$, corrected for multiple comparisons) were colored.

lies in the use of subject-specific time shifts and acceleration factors which appear as random effects of our statistical model. The acceleration factor and time shift associated to a given individual allow to define the subject-specific trajectory of disease progression as an affine reparametrization of the average diseaseprogression trajectory. As a consequence, the progression of a given individual can be easily compared to the estimated average scenario and two individuals can be compared together through the comparisons of their corresponding affine reparametrizations. These subject-specific time shift account for delay (or advance) in disease progression. Using the logistic curves model and the straight lines model, we showed that we were able to distinguish between fast versus slow progressing individuals and early versus late onset individuals. However, our experimental results were based on the a priori knowledge of clinical diagnostic informations and the statistical model we introduced does not allow to determine the sequence in which biomarkers reach pathological levels. Fonteijn et al. proposed such an event-based model in [14]. Finally, our model was described in a Riemannian manifold setting which ensures that the model can be applied to 

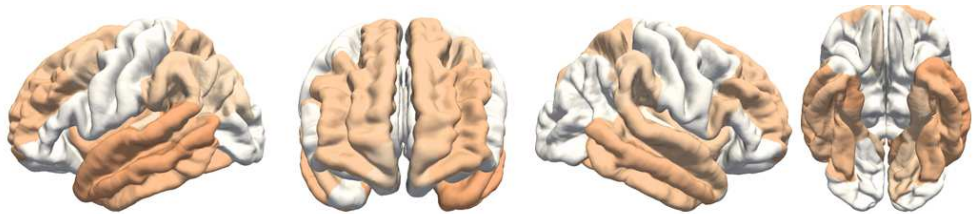

Difference of acceleration factors
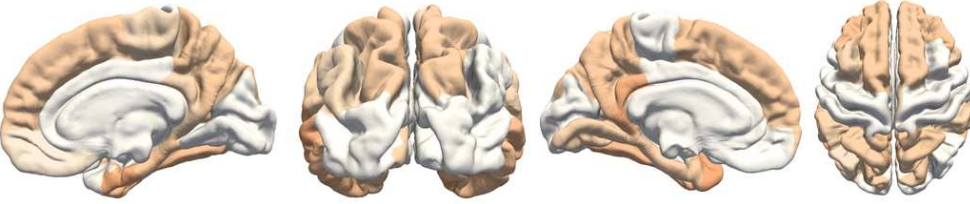

1.1

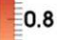

0.4

0

$-0.4$
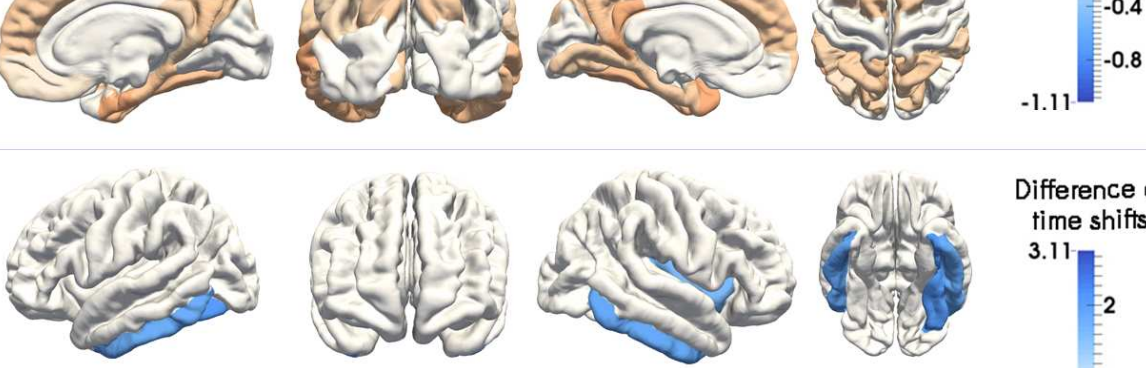

Difference of

time shifts

3.11
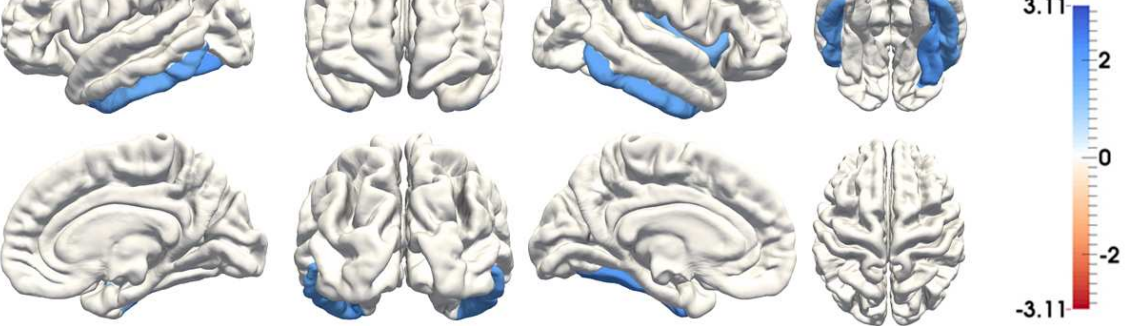

Fig. 3. At the top (respectively bottom) : the difference in averaged acceleration factors (respectively time shifts) between converters MCI and stable MCI is displayed on the cortex. Acceleration factors (and respectively time shifts) were averaged per regions of interest. Only regions where the difference was statistically significant $(p<0.05$, corrected for multiple comparisons) were colored.

data of varying complexity. This also paves the way for a generalization of this model to a multivariate framework.

Acknowledgments. This work was partially supported by the FMJH (Governement Program: ANR-10-CAMP-0151-02). The research leading to these results has received funding from the program Investissements davenir ANR-10IAIHU-06.

\section{References}

1. Jack Jr, C. R., Knopman, D. S., Jagust, W. J., Shaw, L. M., Aisen, P. S., Weiner, M. W., ... \& Trojanowski, J. Q.: Hypothetical model of dynamic biomarkers of the Alzheimer's pathological cascade. The Lancet Neurology 9(1), 119-128 (2010)

2. Samtani, M. N., Raghavan, N., Shi, Y., Novak, G., Farnum, M., Lobanov, V., ... \& Narayan, V. A.: Disease progression model in subjects with mild cognitive impairment from the Alzheimer's disease neuroimaging initiative: CSF biomarkers predict population subtypes. British Journal of Clinical Pharmacology 75(1), 146-161 (2013) 
3. Delor, I., Charoin, J. E., Gieschke, R., Retout, S., \& Jacqmin, P.: Modeling Alzheimers Disease Progression Using Disease Onset Time and Disease Trajectory Concepts Applied to CDR-SOB Scores From ADNI. CPT Pharmacometrics Syst Pharmacology 2(10), e78 (2013)

4. Yang, E., Farnum, M., Lobanov, V., Schultz, T., Raghavan, N., Samtani, M. N., ... \& DiBernardo, A.: Quantifying the pathophysiological timeline of Alzheimer's disease. Journal of Alzheimer's Disease 26(4), pp. 745-753 (2011)

5. Laird, N. M., \& Ware, J. H.: Random-effects models for longitudinal data. Biometrics, 963-974 (1982)

6. Do Carmo, M. P.: Riemannian geometry. Springer (1992)

7. Datar, M., Muralidharan, P., Kumar, A., Gouttard, S., Piven, J., Gerig, G., ... \& Fletcher, P. T.: Mixed-Effects shape models for estimating longitudinal changes in anatomy. In: Spatio-temporal Image Analysis for Longitudinal and Time-Series Image Data. LNCS, vol. 7570, pp. 76-87. Springer, Heidelberg (2012)

8. Singh, N., Hinkle, J., Joshi, S., \& Fletcher, P. T.: A hierarchical geodesic model for diffeomorphic longitudinal shape analysis. In: Information Processing in Medical Imaging. LNCS, vol. 7917, pp. 560-571. Springer, Heidelberg (2013)

9. Lorenzi, M., Pennec, X., Frisoni, G. B., Ayache, N., \& Alzheimer's Disease Neuroimaging Initiative: Disentangling normal aging from Alzheimer's disease in structural magnetic resonance images. Neurobiol Aging, (2015)

10. Durrleman, S., Pennec, X., Trouvé, A., Braga, J., Gerig, G., \& Ayache, N.: Toward a comprehensive framework for the spatiotemporal statistical analysis of longitudinal shape data. IJCV 103(1), pp. 22-59, (2013)

11. Durrleman, S., Pennec, X., Trouvé, A., Gerig, G., \& Ayache, N.: Spatiotemporal atlas estimation for developmental delay detection in longitudinal datasets. In: Med Image Comput Comput Assist Interv, pp. 297-304. Springer, Heidelberg (2009)

12. Pinheiro, J. C., \& Bates, D. M.: Mixed-effects models in S and S-PLUS. Springer (2000)

13. Lindstrom, M. J., \& Bates, D. M.: Nonlinear mixed effects models for repeated measures data. Biometrics, vol. 46, pp. 673-687 (1990)

14. Fonteijn, H. M., Modat, M., Clarkson, M. J., Barnes, J., Lehmann, M., Hobbs, N. Z., ... \& Alexander, D. C.: An event-based model for disease progression and its application in familial Alzheimer's disease and Huntington's disease. NeuroImage 60(3), pp. 1880-1889 (2012)

15. The Alzheimer's Disease Neuroimaging Initiative, https://ida.loni.usc.edu/

16. Braak H, Braak E.: Staging of Alzheimer's disease-related neurofibrillary changes. Neurobiol Aging 16(3) (1995)

17. Delacourte, A., David, J. P., Sergeant, N., Buee, L., Wattez, A., Vermersch, P., ... \& Di Menza, C.: The biochemical pathway of neurofibrillary degeneration in aging and Alzheimer's disease. Neurology 52(6), vol. 52 (1999)

18. Benzinger, T. L., Blazey, T., Jack, C. R., Koeppe, R. A., Su, Y., Xiong, C., ... \& Morris, J. C.: Regional variability of imaging biomarkers in autosomal dominant Alzheimer's disease. In: Proc Natl Acad Sci U S A.110(47) (2013)

19. Desikan, R. S., Ségonne, F., Fischl, B., Quinn, B. T., Dickerson, B. C., Blacker, D., ... \& Killiany, R. J.: An automated labeling system for subdividing the human cerebral cortex on MRI scans into gyral based regions of interest. Neuroimage. 31(3) (2006) 